\title{
Uso de Sistemas de Gamificação no combate a Evasão de Cursos de Graduação da Área de Exatas
}

\author{
Thales Castro Mendes ${ }^{1}$, Lidiane Teixeira Pereira ${ }^{1}$, Vitória Rios Baranda ${ }^{2}$ \\ Alessandreia Marta de Oliveira Julio ${ }^{1}$, Rodrigo Luis de Souza da Silva ${ }^{1}$ \\ ${ }^{1}$ Departamento de Ciência da Computação \\ 2 Departamento de Artes e Design \\ Universidade Federal de Juiz de Fora \\ Rua José Lourenço Kelmer, s/n - São Pedro - Juiz de Fora - MG - 36036-900
}

\begin{abstract}
The use of gamification is being explored in the context of education in order to raise the engagement of students and support the process of teaching and learning. In most cases, gamification is applied to smaller contexts, like specific classes or tasks. One of the problems with the graduation degrees in the mathematical fields is the high evasion of students due to several distinct factors, which makes your characterization more complex. However, it is believed that one of the factors that impacts the most is the lack of motivation of the student with his graduation. Thus, this paper proposes to study and analyze the impact of the use of gamification in the reduction of the evasion in the mathematical degrees. To accomplish this task, we proposed an initial study with a Computer Science graduation, where the students will use a web system that shows, in a gamified way, information about the graduation as a whole. One qualitative evaluation was performed, which through a questionnaire, freshman and seniors that made use of the system rated the potential of the application in the increase of the engagement through the graduation. The evaluation was positive and most of the students agreed that the system have potential to rise the engagement of the students and also agreed to recommend the use to further colleagues.
\end{abstract}

Resumo. O uso de gamificação vem sendo explorado no contexto da educação a fim de aumentar o engajamento dos alunos e apoiar o processo de ensino e aprendizagem. Na maioria dos casos, a gamificação é aplicada a contextos menores, como o de tarefas ou disciplinas específicas. Um dos problemas dos cursos de graduação na área de Ciência Exatas é a alta taxa de evasão devido a diversos fatores distintos, o que torna sua caracterização mais complexa. No entanto, acredita-se que um dos fatores com grande impacto é a falta de motivação do aluno no curso. Dessa forma, o presente trabalho se propõe a estudar e analisar o impacto do uso de gamificação na diminuição da evasão nos cursos de Ciências Exatas. Para isso, foi proposto um estudo com o curso de Ciência da Computação, onde os alunos utilizaram um sistema que exibe, de maneira gamificada, suas informações sobre o curso como um todo. Uma avaliação de caráter qualitativo foi proposta. Nesta avaliação calouros e veteranos utilizaram o sistema e avaliaram seu potencial no aumento do engajamento no curso. As avaliações foram positivas e a maioria dos alunos concordou que o sistema teria potencial para aumentar o engajamento entre os alunos e também concordou que recomendaria o uso aos demais colegas do curso. 
VIII Congresso Brasileiro de Informática na Educação (CBIE 2019)

Anais do XXX Simpósio Brasileiro de Informática na Educação (SBIE 2019)

\section{Introdução}

A gamificação pode ser entendida como o uso de mecânicas e dinâmicas de jogos para engajar pessoas, resolver problemas e melhorar o aprendizado, motivando ações e comportamentos em ambientes fora do contexto de jogos [Deterding et al. 2011]. Estudos que utilizam gamificação para apoiar o ensino e aprendizagem encontrados na literatura apresentam, de forma geral, resultados satisfatórios em relação ao engajamento e aprendizado dos alunos em tarefas e disciplinas específicas [Hamari et al. 2014, Iosup and Epema 2014, Freitas et al. 2016, Barata et al. 2013].

Um aspecto a ser explorado neste artigo está relacionado ao engajamento dos alunos em relação ao seu curso e o potencial deste engajamento para melhorar o desempenho no curso e reduzir a evasão. Vários cursos da área de Ciências Exatas sofrem com o problema de evasão escolar. Conforme apresentado em [Souza et al. 2012], nas disciplinas da área de Ciências Exatas, muitos alunos, de todos os níveis de ensino, enfrentam dificuldades no acesso ao conhecimento. Possivelmente este fenômeno está relacionado à falta de pré-requisitos para acompanhar as disciplinas, dentre as quais destacam-se as disciplinas da área de matemática. A pesquisa aponta que em 2005 o índice de abandono nos cursos de Ciências Exatas chegou a 44\%. Um estudo mais recente [Ciribelli 2015], mostra que em 2014, das 245 vagas ofertadas para o curso de Bacharelado Interdisciplinar em Ciências Exatas, a evasão chegou a 40\% (95 alunos).

Essa evasão se dá em parte pela dificuldade que os alunos enfrentam nas disciplinas iniciais do curso. Uma questão de pesquisa levantada neste artigo é que enfatizar o uso de estratégias de gamificação pode aumentar o engajamento dos alunos em relação ao seu curso, em especial os alunos dos períodos iniciais.

Pesquisas recentes apontam que sistemas gamificados focados em um grupo específico de pessoas, se bem projetados, tem como potencial aumentar o engajamento desse grupo facilitando a superação, em grupo, de objetivos individuais [Morschheuser et al. 2017]. Outro ponto a ser analisado é que, acredita-se que um sistema que reúna alunos mais experientes a outros de períodos iniciais pode colaborar para que os últimos tenham maior interesse e, por consequência, melhor aproveitamento em seu curso, diminuindo desta forma a evasão nos períodos iniciais.

O presente trabalho propõe o uso de gamificação para aumentar o engajamento de alunos em relação ao curso e entre si, promovendo um maior engajamento entre veteranos e alunos em períodos iniciais utilizando para tal mecânicas e dinâmicas de jogos em um sistema Web, denominado SigComp. Para tanto, este artigo está organizado como a seguir. A Seção 2 apresenta os trabalhos relacionados e a Seção 3, o sistema proposto. A Seção 4 apresenta uma análise qualitativa do SigComp seguida de uma análise dos resultados na Seção 5. A Seção 6 se refere às ameaças à validade enquanto as considerações finais são discutidas na Seção 7.

\section{Trabalhos Relacionados}

Nesta seção são descritos alguns trabalhos relacionados à proposta deste artigo. Vale mencionar que a maioria é utilizada para aumentar o engajamento de alunos através de iniciativas gamificadas para determinadas tarefas e/ou disciplinas.

Em [Kuo and Chuang 2016], uma plataforma de gamificação foi desenvolvida 
VIII Congresso Brasileiro de Informática na Educação (CBIE 2019)

Anais do XXX Simpósio Brasileiro de Informática na Educação (SBIE 2019)

para promover conteúdo educacional, sendo acessada por estudantes do ensino superior, visitantes e profissionais na indústria. Dentre as principais conclusões, foi constatado, através de uma consulta online aos participantes, que incentivos gráficos, atividades temáticas gamificadas e o fórum de discussão são os três mais importantes fatores de engajamento e permanência dos participantes no curso. Estas conclusões podem dar suporte à criação de sistemas baseados em gamificação com maior poder de atrair e manter os participantes na plataforma desenvolvida, sendo esta característica de especial interesse para sistemas educacionais e para sistemas de e-commerce e redes sociais.

Um experimento de longa duração (3 anos) envolvendo a aplicação de técnicas de gamificação em cursos de graduação e mestrado foi apresentado em [Iosup and Epema 2014]. Neste experimento, duas disciplinas, uma de graduação e outra de mestrado, receberam elementos de gamificação e foram aplicadas a um total de 450 alunos durante este período. Os autores atribuem à gamificação o aumento do número de alunos aprovados nas disciplinas e do engajamento dos alunos nestes cursos.

O trabalho apresentado em [Freitas et al. 2016] também apresenta uma abordagem de gamificação de disciplina bem sucedida. Os autores partiram do pressuposto que atividades com gamificação tem mais chance de serem bem sucedidas se envolverem alunos ambientados com dinâmicas de jogos. Neste trabalho, 73,08\% dos alunos jogaram mais de 10 jogos eletrônicos e em torno de $50 \%$ dos alunos ainda jogavam de 1 a 6 horas por semana. Os autores concluíram que o uso de gamificação aumentou a dedicação dos alunos em relação à disciplina avaliada.

Resultados semelhantes foram encontrados em [Barata et al. 2013]. Neste trabalho, uma disciplina foi analisada por 5 anos, sendo os três primeiros sem gamificação e os dois últimos com. Os dados destes 5 anos foram analisados e chegou-se a conclusão que a disciplina, quando gamificada, trouxe benefícios relacionados à participação dos alunos nas atividades propostas e na proatividade dos mesmos.

Em [Wiener and de Campos 2018] foi proposto o Colligo App, um aplicativo que permite o uso de técnicas de gamificação em sala de aula para auxiliar o ensino e aprendizagem em disciplinas diversas. O aplicativo busca crescimento técnico e pessoal do aluno e faz uso de elementos de pontuação, rankings, emblemas e cartas. Uma avaliação preliminar em um cenário de ensino superior foi realizada e por meio dela foi possível observar que os usuários se mostraram satisfeitos sobre as funcionalidades do aplicativo e a maioria concordou que ele poderia estimular a participação dos alunos nas aulas.

Há trabalhos na literatura que ao invés de proporem disciplinas gamificadas, focam no uso de gamificação como apoio à disciplinas em curso como em [Knutas et al. 2014]. Neste trabalho foi proposto um sistema colaborativo de discussão com elementos de gamificação para apoiar a condução de uma determinada disciplina. $\mathrm{O}$ experimento foi realizado com 249 alunos e destes, 61 participaram de sua avaliação. Os resultados apontaram uma melhora significativa na colaboração entre os alunos, ressaltando o envolvimento de alunos mais experientes em apoio aos alunos novatos.

Vale ressaltar que nem todas as iniciativas que fazem uso de gamificação apresentam resultados satisfatórios como apontado em [Berkling and Thomas 2013]. Neste trabalho, uma proposta de gamificação de uma disciplina Engenharia de Software foi apresentada. O objetivo do trabalho era aumentar o engajamento e motivação dos alunos 
através de métodos de flexibilização da velocidade de aprendizado e diferentes ênfases nas escolhas do que o aluno poderia estudar em um dado momento. Ao contrário do inicialmente esperado pelos autores, no estudo qualitativo, os alunos não acharam interessante a gamificação do curso. Uma das críticas dos alunos estava relacionada à premissa de que alunos de computação, em sua maioria, são jogadores e isso não se mostrou verdade na universidade onde o experimento foi realizado. Os alunos relataram neste trabalho que se sentiam mais confortáveis com métodos tradicionais de ensino.

O sistema proposto neste artigo difere dos citados nesta seção por propor a utilização de elementos de gamificação em um curso como um todo e não em uma disciplina em particular. A ideia central desta iniciativa é avaliar o quanto um sistema de gamificação desta natureza pode ser útil no aumento do engajamento dos alunos entre si e em relação ao curso. No contexto da pesquisa realizada, não foram encontrados na literatura sistemas com proposta similar.

\section{SigComp}

O SigComp visa, por meio da aplicação de técnicas de gamificação, diminuir a evasão e melhorar o desempenho dos alunos do curso de Ciência da Computação. Caso se prove eficiente, a intenção é expandir para os outros cursos da mesma instituição de ensino.

O sistema possui uma série de funcionalidades para aumentar o engajamento dos alunos do curso, entre elas, o quadro de medalhas, o painel de pontuação e atributos, o ranking (leaderboard) e a lista de amigos. Tais funcionalidades foram elaboradas e implementadas de forma a satisfazer as três necessidades inatas para gerar uma motivação intrínseca descritas em [Groh 2012]:

- Relacionamentos: necessidade universal de interagir e se conectar com outros;

- Competência: necessidade universal de ser eficiente e dominar um problema num dado ambiente;

- Autonomia: necessidade universal de ter controle sobre sua vida.

A necessidade de relacionamento está ligada à vontade inata de se conectar com os outros. Para satisfazer essa necessidade, está em curso a implementação da funcionalidade do fórum de dúvidas e interação dos alunos. Dessa forma, eles terão uma motivação intrínseca de ajudar os seus colegas e conversar sobre assuntos em geral, fazendo com que todos se sintam mais integrados. Há também uma lista de amigos, permitindo a visualização do perfil dos amigos do usuário, gerando uma certa competitividade pelas medalhas mais raras e atributos maiores.

Vale ainda destacar que todos os usuários do sistema são alunos do mesmo curso e pressupõe-se que possuam muitos interesses em comum, podendo ser vistos como uma meaningfull community [Groh 2012]. Dessa forma, a visualização das medalhas no perfil de um usuário é feita por outros usuários que entendem seu significado e valor.

A necessidade de competência está associada à sensação de querer ser bom no que se faz. Ainda segundo [Groh 2012], isso pode ser alcançado explicitando ao usuário desafios interessantes em uma forma clara, visual, com regras e objetivos bem definidos. Para estimular essa necessidade foram criados o quadro de medalhas, os atributos e o leaderboard. Essas são partes do sistema que visam valorizar o empenho que cada um coloca nas suas tarefas usuais e prover um feedback claro dos seus resultados. 
VIII Congresso Brasileiro de Informática na Educação (CBIE 2019)

Anais do XXX Simpósio Brasileiro de Informática na Educação (SBIE 2019)

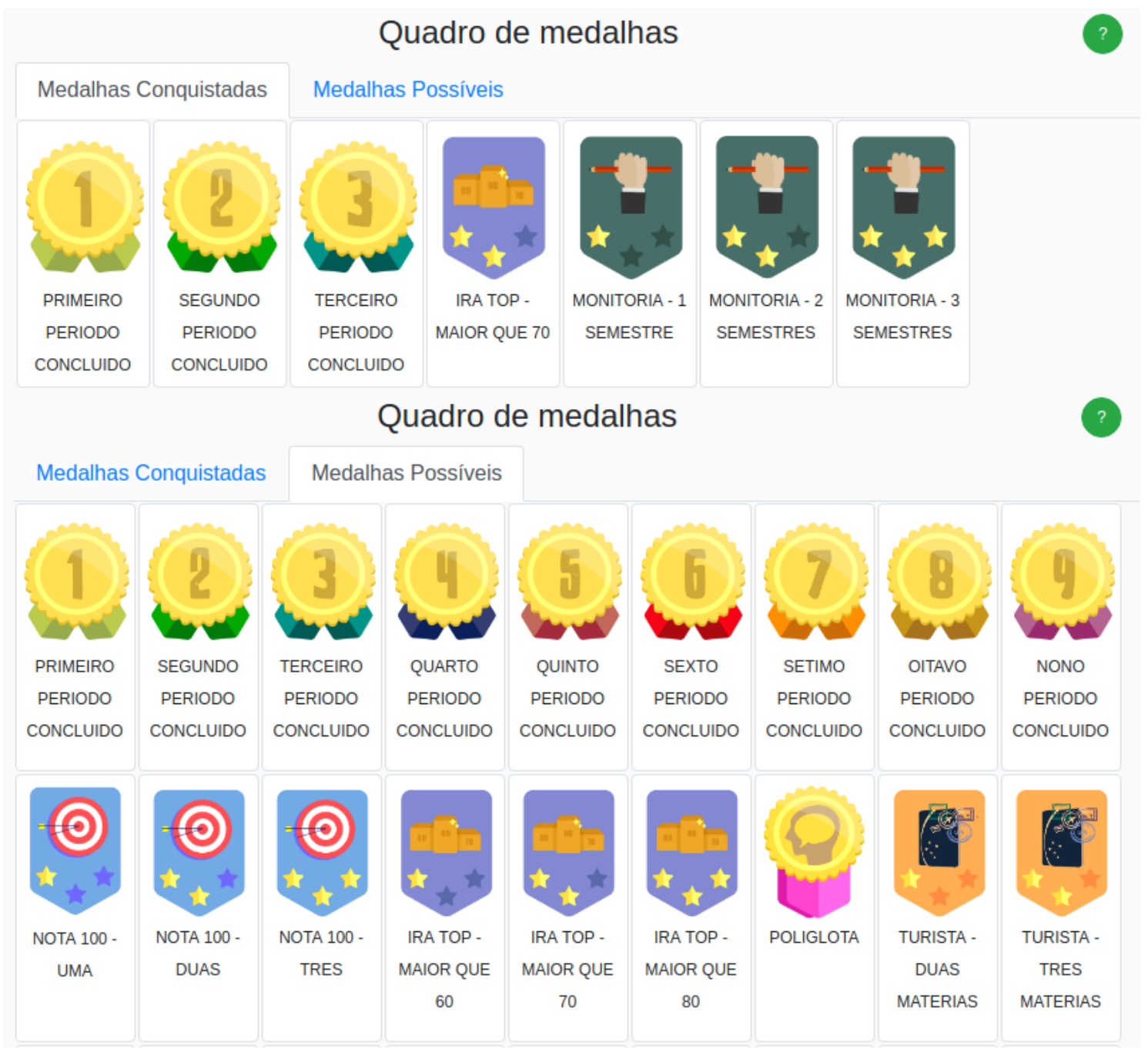

Figura 1. Medalhas conquistadas por um aluno fictício e medalhas possíveis.

Quando o aluno atinge certos requisitos, ele recebe uma medalha no seu perfil, como exemplificado na Figura 1. Para o usuário conhecer as metas alcançáveis e assim se motivar para atingi-las, foi criada a aba Medalhas Possíveis no quadro de medalhas, dentre elas, possuir Índice de Rendimento Acadêmico (IRA) maior que 70, participar de bolsa de Iniciação Científica ou de Monitoria e obter uma ou mais notas 100 no curso. É possível também visualizar as medalhas dos amigos através da visita ao perfil dos mesmos, gerando assim uma valorização das medalhas mais difíceis de alcançar e um aspecto de competição entre os alunos, o que pode ser uma motivação extra.

Para incentivar o aluno a alcançar melhores notas, foi criado um sistema fictício de atributos como em um jogo de role-playing game (RPG), em que o desempenho do aluno em certas disciplinas aumenta sua pontuação em atributos específicos. Por exemplo, boas notas em disciplinas como Estruturas de Dados podem aumentar sua Destreza, enquanto boas notas em Cálculo aumentam sua Força.

Com o intuito de criar um espírito de competição foi implementado um leaderboard, onde os alunos com maior IRA são mostrados na página inicial. Porém somente são exibidos os alunos que explicitamente concordaram em ter seus nomes divulgados. 
VIII Congresso Brasileiro de Informática na Educação (CBIE 2019)

Anais do XXX Simpósio Brasileiro de Informática na Educação (SBIE 2019)

Para promover uma competitividade entre os alunos é necessário que exista uma forma de comparação dos seus resultados. Para isso foi implementada a lista de amigos. Através dela, um aluno pode entrar no perfil de quem é seu amigo e verificar seu desempenho e suas conquistas atuais.

Por último, acerca da necessidade de autonomia, que é a ânsia por liberdade, foi considerado que a mesma é inerente ao sistema, já que tudo que afeta o resultado de um aluno está relacionado com suas próprias conquistas.

Também em [Schell 2011], o atendimento às necessidades inatas de relacionamento, competência e autonomia, é apontado como essencial para sistemas de gamificação bem sucedidos. Acredita-se que as funcionalidades implementadas e anteriormente descritas estejam suprindo tais necessidades.

\section{Estudo Qualitativo}

Neste estudo, pretende-se caracterizar o apoio existente através do SigComp e o uso de elementos de gamificação no engajamento e desempenho dos alunos do curso de Ciência da Computação.

Para avaliar se o SigComp atingiu os seus objetivos, foi realizado um estudo visando responder às seguintes questões de pesquisa $(\mathrm{QP})$ :

- QP1: Um sistema gamificado que utiliza elementos de design de jogos (medalhas, ranking, ...) no âmbito geral do curso pode contribuir com o aumento do engajamento e desempenho dos alunos no curso de Ciência da Computação?

- QP2: Um sistema que promova a interação entre alunos de períodos iniciais (calouros) com os veteranos pode colaborar para aumentar o engajamento dos calouros no curso de Ciência da Computação?

Inicialmente o convite para participação do estudo foi enviado para aproximadamente 40 alunos do curso de Ciência da Computação. Destes convidados, 21 demonstraram interesse mas um tinha menos de 18 anos e não pode participar, resultando em 20 alunos que efetivamente participaram do estudo. Os participantes tinham idade variando de 18 a 23 anos, sendo 10 alunos do segundo período e os outros 10 de períodos mais altos. Foram identificados 18 alunos do sexo masculino e duas alunas do sexo feminino.

O estudo consistia na utilização do SigComp e na identificação de suas características e execução de algumas operações práticas. Para tanto, um guia foi enviado aos alunos juntamente com um certificado fictício e o link de um formulário de avaliação. $\mathrm{O}$ formulário enviado continha perguntas pessoais como idade, ano de ingresso e questões relacionadas a jogos eletrônicos. Em relação ao sistema, foram enviadas afirmações a serem avaliadas de acordo com a escala de Likert de cinco pontos [Likert 1932] devido à sua adequação para medir a opinião dos participantes e facilidade de análise dos resultados. As afirmações incluíam informações sobre facilidade de utilização do sistema e sobre quão úteis seriam suas funcionalidades. Ao final, havia uma questão discursiva para que o participante informasse quais funcionalidades poderiam ser adicionadas ao sistema para aumentar o engajamento dos alunos entre si e em relação ao curso.

\section{Resultados e Discussão}

Uma das questões do formulário de avaliação tratava do interesse dos participantes por jogos eletrônicos. Todos os participantes do estudo responderam positivamente. Em relação 
VIII Congresso Brasileiro de Informática na Educação (CBIE 2019)

Anais do XXX Simpósio Brasileiro de Informática na Educação (SBIE 2019)

à plataforma de jogos, a grande maioria utiliza computadores (19 alunos), 10 utilizam celular e 8 videogame. A soma é maior que o número total de participantes pois o aluno poderia selecionar mais de um dispositivo que utiliza para jogos. Em relação ao tempo gasto semanalmente com jogos, as respostas foram bem divididas. As possibilidades eram "não costuma jogar semanalmente" ( 3 alunos), "menos de 5 horas" (6 alunos), "entre 5 e 10 horas" (7 alunos) e "mais de 10 horas" (4 alunos).

Em relação às características do SigComp, as respostas estão sumarizadas na Tabela 1. Pode-se observar que a maioria dos alunos achou a interface do sistema intuitiva e de fácil utilização (19 alunos concordaram total ou parcialmente).

Sobre as características relacionadas a sistemas de gamificação, a percepção dos alunos de forma geral foi positiva. O quadro de medalhas foi visto como um fator motivador por 17 alunos e o ranking dividiu opiniões. Doze alunos concordaram que a presença do ranking baseado no índice de rendimento acadêmico seria um fator motivador, mas 6 ficaram neutros e 2 alunos discordaram. Resultado semelhante foi encontrado quando a afirmação era se um ambiente virtual de cunho competitivo poderia motivar o aluno a ter um melhor desempenho. Neste item, 15 alunos concordaram com a afirmação, mas 4 ficaram neutros e 1 aluno discordou. A grande maioria dos alunos concordou que o SigComp teria potencial para aumentar o engajamento entre os alunos.

Em relação às funcionalidades planejadas como próximas atividades a serem desenvolvidas, 17 alunos consideraram que um fórum de dúvidas seria útil e todos os 20 alunos concordaram que um mural de oportunidades seria relevante para o curso. A recomendação de cadastro no sistema também foi unânime entre os alunos.

Em relação à questão discursiva sobre possíveis funcionalidades do sistema para aumentar o engajamento dos alunos entre si e em relação ao curso, as respostas foram as mais diversas. No que diz respeito à opinião dos alunos de segundo período, não foi possível identificar uma tendência. Um dos alunos indicou que notícias sobre oportunidades como bolsas de iniciação científica e outros projetos acadêmicos seriam interessantes. Vale mencionar que esse aspecto já está em desenvolvimento no contexto do mural de oportunidades. Houve a indicação por parte de um aluno de incluir jogos relacionados a temas abordados em disciplinas como forma de aprender conteúdos de forma lúdica. $\mathrm{Na}$ mesma linha, outro aluno indicou que poderiam ser implementadas "missões" envolvendo problemas de programação, com recompensas para os alunos que as concluírem. Dois alunos sugeriram incluir um ranking geral dos índices de rendimento acadêmico. Esta opção foi cogitada quando da concepção do sistema, mas foi considerada possivelmente contraproducente por expor para um aluno mal posicionado, sua posição em relação aos demais, sendo esse um possível fator desmotivador.

Os veteranos, além de algumas correções pontuais, indicaram direções que julgaram interessantes para o sistema. Dois alunos indicaram, na mesma linha dos alunos de segundo período, a possibilidade de incluir um ranking geral. A possibilidade de incluir um currículo pessoal foi levantada por um aluno, onde seria possível indicar conhecimentos e interesses, tanto profissionais quanto pessoais. Na visão do aluno, esta funcionalidade poderia auxiliar os que ainda não tem nenhuma experiência de trabalho ou estágio a indicar seus interesses dentro do curso. Dois alunos indicaram que o fórum de dúvidas poderia ter uma categoria livre, onde os alunos poderiam marcar atividades não 
VIII Congresso Brasileiro de Informática na Educação (CBIE 2019)

Anais do XXX Simpósio Brasileiro de Informática na Educação (SBIE 2019)

Tabela 1. Resultados da avaliação qualitativa

\begin{tabular}{|l|c|c|c|c|c|}
\hline \multicolumn{1}{|c|}{ Afirmação } & DC & DP & N & CP & CC \\
\hline A interface do sistema é intuitiva e de fácil utilização. & 0 & 0 & 2 & 7 & 11 \\
\hline $\begin{array}{l}\text { A disponibilização de links para redes sociais e profissionais } \\
\text { (Facebook, Instagram, Linkedin etc) do aluno é uma funcio- } \\
\text { nalidade útil no sistema. }\end{array}$ & 0 & 1 & 2 & 6 & 11 \\
\hline $\begin{array}{l}\text { O quadro de medalhas baseado nas conquistas do aluno den- } \\
\text { tro do curso é uma funcionalidade que motiva o aluno a obter } \\
\text { melhores resultados. }\end{array}$ & 0 & 0 & 3 & 11 & 6 \\
\hline $\begin{array}{l}\text { O ranking através do IRA é uma funcionalidade que motiva o } \\
\text { aluno a obter melhores resultados. }\end{array}$ & 0 & 2 & 6 & 9 & 3 \\
\hline $\begin{array}{l}\text { Um ambiente virtual de cunho competitivo (uso de ranking, } \\
\text { medalhas, barras de progresso etc) pode motivar o aluno a ter } \\
\text { um melhor desempenho acadêmico em seu curso. }\end{array}$ & 0 & 1 & 4 & 11 & 4 \\
\hline $\begin{array}{l}\text { Um ambiente como o SigComp pode aumentar o engajamento } \\
\text { entre os alunos. }\end{array}$ & 0 & 0 & 4 & 10 & 6 \\
\hline $\begin{array}{l}\text { Uma funcionalidade planejada no SigComp é um fórum de } \\
\text { dúvidas. Essa funcionalidade pode ser útil para que os alunos } \\
\text { tirem dúvidas entre si. }\end{array}$ & 0 & 0 & 3 & 6 & 11 \\
\hline $\begin{array}{l}\text { Uma funcionalidade planejada no SigComp é a inclusão de } \\
\text { um mural de oportunidades. Essa funcionalidade pode ser } \\
\text { relevante para os alunos do curso. }\end{array}$ & 0 & 0 & 0 & 4 & 16 \\
\hline $\begin{array}{l}\text { Você recomendaria o cadastro no SigComp a um colega do } \\
\text { curso de Ciência da Computação. }\end{array}$ & 0 & 0 & 0 & 9 & 11 \\
\hline
\end{tabular}

acadêmicas como participação em jogos de tabuleiros e outros hobbies. Um dos alunos que fez esta indicação, salientou que alguns alunos com menor comprometimento poderiam aumentar sua interação com os demais e com o curso se identificassem colegas com interesses semelhantes aos seus. Finalmente um dos alunos indicou a possibilidade de serem atribuídas medalhas relacionadas a uma determinada área do curso e à medida que o aluno fosse completando as disciplinas daquele perfil subiria de nível naquela área.

\section{Ameaças à Validade}

Durante o planejamento deste estudo, buscou-se minimizar ameaças que pudessem impactar ou limitar a validade dos resultados obtidos [Wohlin et al. 2000]. No entanto, não é possível garantir que tais ameaças não tenham afetado os resultados. Desta forma, as ameaças identificadas no contexto deste estudo são descritas a seguir.

O estudo foi executado no local e horário mais conveniente para cada participante, em função da disponibilidade dos mesmos. Isto pode ter influenciado os resultados, já que não é possível confirmar que as circunstâncias eram as mesmas nas ocasiões em que cada participante executou o estudo. Contudo, o mesmo roteiro e o mesmo ambiente foi utilizado com a intenção de minimizar esta ameaça.

O entendimento dos participantes sobre as questões dos formulários é diretamente influenciado pela forma como foram elaboradas. A análise dos instrumentos utilizados (inclusive os formulários) a partir de um estudo piloto visou reduzir esta interferência. 
VIII Congresso Brasileiro de Informática na Educação (CBIE 2019)

Anais do XXX Simpósio Brasileiro de Informática na Educação (SBIE 2019)

O tamanho da amostra é limitado, o que não é ideal do ponto de vista estatístico. Desta forma, os resultados do estudo não são conclusivos: somente fornecem indícios. No entanto, o número de participantes superior a 20 é considerado alto [Juristo and Moreno 2013], o que aumenta a validade estatística das conclusões obtidas.

\section{Conclusão e Trabalhos Futuros}

Este artigo apresentou um sistema de gamificação com o objetivo de aumentar o engajamento de alunos do curso de Ciência da Computação, potencialmente colaborando para o aumento do desempenho destes alunos e a diminuição dos índices de evasão do curso. $\mathrm{O}$ sistema proposto adiciona elementos de gamificação (sistemas de medalhas, ranking, lista de amigos, entre outros) ao curso como um todo e usa como entrada os dados disponíveis dos alunos para aumentar o engajamento.

A avaliação do sistema foi realizada através de um estudo qualitativo onde os alunos eram guiados pelo sistema identificando suas características e realizando algumas operações práticas para entender suas funcionalidades. Após a aplicação do estudo foi feita uma avaliação através do preenchimento de um formulário. Foi realizada uma análise qualitativa das respostas onde foi possível constatar que os alunos aprovaram a utilização do sistema para o que ele se propunha. Diante da aceitação dos alunos, pretende-se realizar melhorias nas funcionalidades do sistema e uma análise dos índices de evasão do curso antes e depois da aplicação do sistema proposto ao longo dos próximos semestres.

Através do formulário de avaliação foram coletadas informações sobre possíveis funcionalidades a serem adicionadas ao sistema que estão sendo estudadas para implementação futura. Um exemplo é o Mural de Oportunidades, onde os professores poderão criar anúncios para seus projetos a fim de convocar alunos. Foi considerada a opção do professor criar pré-requisitos pautados no sistema de atributos, como por exemplo um projeto que é necessário pelo menos 30 de Destreza para inscrição, criando um interesse por parte dos alunos em conseguir notas altas para possuir os atributos necessários.

Para a realização de uma avaliação mais aprofundada, está em fase de implementação a inclusão da ferramenta Google Analytics ${ }^{1}$, onde será possível estudar mais sobre o comportamento dos usuários, possibilitando observar métricas como o tempo gasto no sistema em cada visita, as páginas mais acessadas, em quais datas houve um maior fluxo de usuários entre outras. Assim, esses dados serão usados para criar uma experiência otimizada a fim de fomentar ainda mais o uso do sistema. Espera-se também que tais métricas auxiliem tanto na elaboração das atividades quanto na coleta de dados em um estudo experimental previsto como trabalho futuro.

\section{Referências}

Barata, G., Gama, S., Jorge, J., and Gonçalves, D. (2013). Improving participation and learning with gamification. In Proceedings of the First International Conference on gameful design, research, and applications, pages 10-17. ACM.

Berkling, K. and Thomas, C. (2013). Gamification of a software engineering course and a detailed analysis of the factors that lead to it's failure. In International Conference on Interactive Collaborative Learning, pages 525-530. IEEE.

\footnotetext{
${ }^{1}$ https://analytics.google.com/analytics/web/[Online; acessado em: 01/07/2019]
} 
VIII Congresso Brasileiro de Informática na Educação (CBIE 2019)

Anais do XXX Simpósio Brasileiro de Informática na Educação (SBIE 2019)

Ciribelli, B. C. N. (2015). Retenção e evasão escolares no bacharelado interdisciplinar em ciências exatas da universidade federal de juiz de fora. Master's thesis, Universidade Federal de Juiz de Fora.

Deterding, S., Dixon, D., Khaled, R., and Nacke, L. (2011). From game design elements to gamefulness: defining "gamification". In Proceedings of the 15th International Academic MindTrek Conference: Envisioning Future Media Environments, pages 915. ACM.

Freitas, S., Lima, T., Canedo, E., and Costa, R. L. (2016). Gamificação e avaliação do engajamento dos estudantes em uma disciplina técnica de curso de graduação. In Anais do XXVII Simpósio Brasileiro de Informática na Educação, volume 27, page 370.

Groh, F. (2012). Gamification: state of the art definition and utilization. Institute of Media Informatics Ulm University, 39:31.

Hamari, J., Koivisto, J., and Sarsa, H. (2014). Does gamification work? a literature review of empirical studies on gamification. In Proceedings of the 47th Hawaii International Conference on System Sciences, volume 14, pages 3025-3034.

Iosup, A. and Epema, D. (2014). An experience report on using gamification in technical higher education. In Proceedings of the 45th ACM Technical Symposium on Computer Science Education, pages 27-32. ACM.

Juristo, N. and Moreno, A. M. (2013). Basics of software engineering experimentation. Springer Science \& Business Media.

Knutas, A., Ikonen, J., Nikula, U., and Porras, J. (2014). Increasing collaborative communications in a programming course with gamification: a case study. In Proceedings of the 15th International Conference on Computer Systems and Technologies, pages 370-377. ACM.

Kuo, M.-S. and Chuang, T.-Y. (2016). How gamification motivates visits and engagement for online academic dissemination-an empirical study. Computers in Human Behavior, $55: 16-27$.

Likert, R. (1932). A technique for the measurement of attitudes. Archives of psychology.

Morschheuser, B., Riar, M., Hamari, J., and Maedche, A. (2017). How games induce cooperation? a study on the relationship between game features and we-intentions in an augmented reality game. Computers in human behavior, 77:169-183.

Schell, J. (2011). The pleasure revolution: why games will lead the way. GoogleTechTalks Std Proceedings.

Souza, C. T., Silva, C., and Gessinger, R. M. (2012). Um estudo sobre evasão no ensino superior do brasil nos últimos dez anos. In Congresos CLABES.

Wiener, A. and de Campos, A. (2018). Colligo app: gamificação em sala de aula. In Anais dos Workshops do Congresso Brasileiro de Informática na Educação, volume 7, page 272 .

Wohlin, C., Runeson, P., Höst, M., Ohlsson, M. C., Regnell, B., and Wesslén, A. (2000). Experimentation in Software Engineering: An Introduction. Kluwer Academic Publishers, Norwell, MA, USA. 